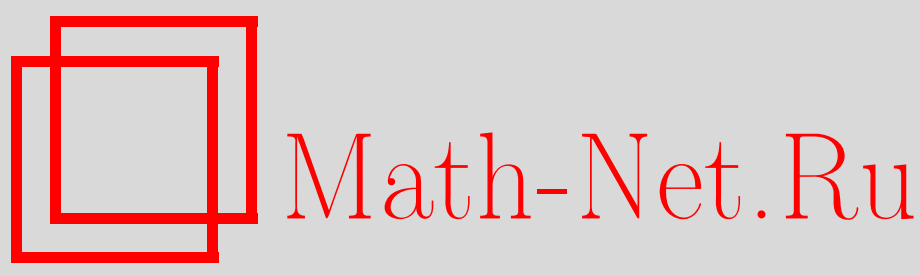

А. А. Карацуба, О дробных долях быстрорастущих функций, Изв. РАН. Сер. матем., 2001, том 65, выпуск 4, 89-110

DOI: https://doi.org/10.4213/im349

Использование Общероссийского математического портала Math-Net.Ru подразумевает, что вы прочитали и согласны с пользовательским соглашением

http: //www.mathnet.ru/rus/agreement

Параметры загрузки:

IP : 54.89 .56 .158

26 апреля 2023 г., $16: 19: 16$ 
УДК 511

\title{
А. А. Карацуба
}

\section{О дробных долях быстрорастущих функций}

\author{
Изучается поведение дробных долей функций вида $\alpha \exp \left(\left[\log ^{c} x\right] \log x\right)$, где \\ $\alpha$ - вещественное алгебраическое число степени $n \geqslant 2$ и $c$ - произвольное поло- \\ жительное число, меньшее единицы. \\ Библиографиял: 12 наименований.
}

\section{§1. Постановка задачи. Формулировка основного результата}

В [1] автор оценил тригонометрическую сумму $S(f)$ следующего вида:

$$
S(f)=\sum_{x \leqslant X} \exp (2 \pi i m f(x)),
$$

где $m \neq 0, m$ - целое число,

$$
f(x)=\exp \left(c_{1} \log ^{\gamma} x\right)
$$

$c_{1}$ и $\gamma$ - абсолютные положительные константы, причем $1<\gamma<3 / 2$, и, в качестве следствия оценки, получил теорему о равномерном распределении дробных долей $f(x)$.

Более точно эти результаты формулируются так (см. также [2, с. 72] и [3, c. 103]):

ПРЕДЛОЖЕНИЕ 1. Пусть $c_{1}>0$ - произвольное фиксированное число, $\gamma-$ константа, $1<\gamma<3 / 2, \quad m$ - иелое число, $m \neq 0, \quad X \geqslant 1$,

$$
\begin{aligned}
f(x) & =\exp \left(c_{1} \log ^{\gamma} x\right) \\
S=S(f) & =\sum_{x \leqslant X} \exp (2 \pi i m f(x)) .
\end{aligned}
$$

Если $0<|m| \leqslant \exp \left((\log X)^{3-2 \gamma-\varepsilon}\right)$, әде $0<\varepsilon<3-2 \gamma$, mо

$$
|S| \leqslant c_{2} X \exp \left(-c_{3}(\log X)^{3-2 \gamma}\right)
$$

əде $c_{2}=c_{2}(\varepsilon)>0, c_{3}=c_{3}(\varepsilon)>0$. 
ПРЕДЛОЖЕНИЕ 2. Пусть $0<\sigma \leqslant 1$ u $D(\sigma)$ - количество чисел ряда $x=$ $1,2, \ldots, X$ с условием $\{f(x)\}<\sigma$, и пусть

$$
D(\sigma)=\sigma X+\lambda(X) .
$$

Если $f(x)$ удовлетворяет условиям (1), то

$$
\lambda(X)=O\left(X \exp \left(-c_{4}(\log X)^{3-2 \gamma}\right)\right)
$$

где $c_{4}>0$ и постоянная в знаке $O$ зависят только от $\gamma$.

Следствием последнего утверждения является теорема о разрешимости диофантова неравенства (в натуральных числа $x$ ):

При любом вещественном числе $\xi$ выполняется неравенство

$$
\min _{x \leqslant X}\|f(x)-\xi\| \leqslant c_{5} \exp \left(-c_{6}(\log X)^{3-2 \gamma}\right)
$$

где $c_{5}=c_{5}(\gamma)>0, \quad c_{6}=c_{6}(\gamma)>0$ и символ $\|\omega\|$ означает расстояние от вещественного числа $\omega$ до ближсайшего челого числа.

Такое же утверждение справедливо для $\alpha f(x)$ при любом отличном от нуля вешественном числе $\alpha$. Легко видеть, что $f(x)$ при $x \rightarrow+\infty$ стремится $\mathrm{K}+\infty$ быстрее любого многочлена от $x$.

Вопросы о поведении дробных долей вещественнозначных функций и, в частности, вопросы о разрешимости неравенств вида (2) в натуральных числах $x$ с вещественнозначной функцией $f(x)$ были поставлены и изучались в работах [4]-[8]. При доказательстве (2) применялся метод Виноградова оценок тригонометрических сумм с многочленом растущей степени в экспоненте.

В настоящей статье получен результат типа неравенства (2) с функцией $f(x)$, растущей быстрее, чем (1).

Имеет место следующая

ТЕОРЕМА. Пусть $\alpha$ - произвольное вещественное алгебраическое число степени не меньше 2,

$$
f(x)=\alpha \exp \left(\left[\log ^{c} x\right] \log x\right)
$$

где константа с удовлетворяет неравенствам $0<c<1 u\left[\log ^{c} x\right]$ есть иелая часть $\log ^{c} x$. Тогда существуют число $X_{1}=X_{1}(c)>0$ и постоянные $c_{1}>0, c_{2}>0$ такие, что при $X \geqslant X_{1}$ и при любом вещественном числе $\xi$ количество решений неравенства

$$
\|\xi-f(x)\| \leqslant \exp \left(-c_{1} \log ^{1-c} X\right)
$$

в иельх положительных числах $x, x \leqslant X$, не меньие чем

$$
X \exp \left(-c_{2}\left(\log ^{1-c} X+\log ^{c} X \log \log X\right)\right) .
$$

В частности,

$$
\min _{x \leqslant X}\|\xi-f(x)\| \leqslant \exp \left(-c_{1} \log ^{1-c} X\right) .
$$


Метод, на основе которого доказывается теорема, развит в работах автоpa [9]-[12]. Однако прямо применить результаты [11] или [12] к доказательству сформулированной теоремы нельзя из-за слишком жесткой связи главного и второстепенного параметров, введенных в [11] и [12]. Теорема статьи отвечает на один вопрос профессора О. Штрауха, сотрудника Математического института Словакии.

Выражаю глубокую благодарность профессору О. Штрауху за полезное обсуждение проблем теории диофантовых приближений.

Обозначения. Буквами $c, c_{1}, c_{2}, \ldots$ будем обозначать абсолютные положительные постоянные, причем $0<c<1$, а $c_{1}, c_{2}, \ldots$ в разных формулах могут быть разными; буквами $p, p_{1}, p_{2}, \ldots-$ простые числа; символы $[\omega],\{\omega\},\|\omega\|$ обозначают соответственно целую часть вещественного числа $\omega$, т.е. $[\omega]$ - целое число с условием $[\omega] \leqslant \omega<[\omega]+1$, дробную часть $\omega$, т.е. $\{\omega\}=\omega-[\omega]$, и расстояние от $\omega$ до ближайшего целого числа, т.е. $\|\omega\|=\min (\{\omega\}, 1-\{\omega\}) ;$ запись $A \asymp B$ означает, что $c_{1} A \leqslant B \leqslant c_{2} A$; запись $A \ll B$ означает, что $|A| \leqslant c_{1} B ; \pi(x)-$ количество простых чисел, не превосходящих $x ; X$ - основной растуший параметр, $X \geqslant X_{1}(c)>0 ; \log N \asymp \log X ; n$ и $k$ - натуральные числа, причем $n \asymp \log ^{c} X$, $k \asymp \log ^{c} X ; \theta, \theta_{1}, \ldots-$ - некоторые функции с условием $|\theta| \leqslant 1,\left|\theta_{1}\right| \leqslant 1, \ldots ;$ постоянные в знаках $O$ - абсолютные.

\section{§ 2. Основная лемма}

В этом параграфе доказывается основная лемма 1 об оценке сверху количества решений следующего диофантова уравнения:

$$
x_{1}^{n}+x_{2}^{n}+\cdots+x_{k}^{n}=y_{1}^{n}+y_{2}^{n}+\cdots+y_{k}^{n} .
$$

В уравнении (3) неизвестные $x_{1}, \ldots, x_{k}, y_{1}, \ldots, y_{k}$ принимают значения натуральных чисел специального вида, которые в дальнейшем будут называться $v$-иислами. Построим множество натуральных чисел $V$, отвечающее параметрам $N, n$ и $k$, элементы которого и будут $v$-числами. Для этого при $j=1,2, \ldots, k$ определим числа $X_{j}, Y_{j}$ равенствами

$$
X_{j}=\left(1-\frac{1}{4 k}\right) Y_{j}, \quad Y_{j}=N^{\frac{1}{n}\left(1-\frac{1}{n}\right)^{j-1}} .
$$

Далее, при каждом $j, 1 \leqslant j \leqslant k$, заставим параметр $p_{j}$ принимать значения всех простых чисел из промежутка $\left(X_{j}, Y_{j}\right]$, другими словами, заставим $p_{j}$ пробегать последовательные простые числа с условием

$$
X_{j}<p_{j} \leqslant Y_{j}
$$

ОПРЕДЕЛЕНИЕ. $V$-множеством, отвечающим параметрам $N, n u k$, или, коротко, $V$-множеством, называется множество чисел вида $v=p_{1} p_{2} \ldots p_{k}$.

Легко видеть, что $v \neq v^{\prime}$, если только $p_{j} \neq p_{j}^{\prime}$ хотя бы при одном значении $j$, $1 \leqslant j \leqslant k$. Отсюда, в частности, следует, что количество решений уравнения $x y=z$, где $x$ и $y$ - неизвестные $v$-числа, а $z$ - заданное натуральное число, не 
превосходит $2^{k}$. Далее, если символом $\|V\|$ обозначить количество элементов $V$, т.е. количество всех $v$-чисел, то имеем равенство

$$
\|V\|=\prod_{j=1}^{k}\left(\pi\left(Y_{j}\right)-\pi\left(X_{j}\right)\right) .
$$

Пользуясь асимптотической формулой Валле-Пуссена

$$
\pi(x)=\int_{2}^{x} \frac{d t}{\log t}+O\left(x \exp \left(-c_{1} \sqrt{\log x}\right)\right)
$$

находим

$$
\begin{gathered}
\frac{1}{8 k} \frac{Y_{j}}{\log Y_{j}} \leqslant \pi\left(Y_{j}\right)-\pi\left(X_{j}\right) \leqslant \frac{1}{2 k} \frac{Y_{j}}{\log Y_{j}}, \\
4^{-k} M \leqslant\|V\| \leqslant M
\end{gathered}
$$

где

$$
M=\left(\frac{n}{2 k \log N}\right)^{k}\left(1+\frac{1}{n-1}\right)^{\frac{k(k-1)}{2}} N^{1-\left(1-\frac{1}{n}\right)^{k}} .
$$

В свою очередь, верхняя граница $V_{2}$ и нижняя граница $V_{1}$ изменения $v$ таковы:

$$
V_{1}=\left(1-\frac{1}{4 k}\right)^{k} N^{1-\left(1-\frac{1}{n}\right)^{k}} \leqslant v \leqslant N^{1-\left(1-\frac{1}{n}\right)^{k}}=V_{2}
$$

ЛЕмма 1. Пусть I - количество решений уравнения (3) при условии, что $x_{1}, \ldots, x_{k}, y_{1}, \ldots, y_{k}$ принимают значения $v$-чисел, и пусть $2 n+6<k \ll n$. Тогда для I справедлива следующая оценка:

$$
I \leqslant a(n ; k) N^{2 k-n+\omega}
$$

əде

$$
\begin{gathered}
a(n ; k)=(4 k)^{-(k-2 n-5)(1.5 k+n-41)-(2 n+5)(4 n+9)}, \\
\omega=\omega(n ; k)=n\left(1-\frac{1}{n}\right)^{k-2 n-5}-(k+2 n+4)\left(1-\frac{1}{n}\right)^{k}-1 .
\end{gathered}
$$

ДокАЗАТЕльСтво. 1 . При целом числе $s, 0 \leqslant s \leqslant s_{1}=k-2(n+3)$, символом $I^{(s)}$ обозначим количество решений следуюшего уравнения:

$$
x_{s+1}^{n}+\cdots+x_{k}^{n}=y_{s+1}^{n}+\cdots+y_{k}^{n} .
$$

Здесь $x_{j}$ и $y_{j}, j=s+1, \ldots, k$, принимают значения чисел вида $p_{s+1} \ldots p_{k}$, где, в свою очередь, $p_{j}$ принимают значения всех простых чисел соответственно промежутков $\left(X_{j}, Y_{j}\right]$. Очевидно, что $I^{(0)}=I$. 
2. Для величины $I^{(s)}$ докажем следуюшее основное рекуррентное неравенство:

$$
I^{(s)} \leqslant 5 n H^{2}\left(Y_{s+1}-X_{s+1}\right)^{2(k-s-1)} I^{(s+1)} \prod_{j=2}^{k-s}\left(Y_{s+j}-X_{s+j}\right),
$$

где $H=\left(2 k^{5}\right)^{4}$.

3. Представим $I^{(s)}$ в виде интеграла от соответствующей тригонометрической суммы. Пусть

$$
S(\alpha)=\sum_{X_{s+1}<p_{s+1} \leqslant Y_{s+1}} \cdots \sum_{X_{k}<p_{k} \leqslant Y_{k}} \exp \left(2 \pi i \alpha\left(p_{s+1} \ldots p_{k}\right)^{n}\right) .
$$

Тогда имеем следующее равенство:

$$
I^{(s)}=\int_{0}^{1}|S(\alpha)|^{2(k-s)} d \alpha .
$$

4. Преобразуем $S(\alpha)$. Вводя новые тригонометрические суммы

$$
S\left(\alpha ; p_{s+1}\right)=\sum_{X_{s+2}<p_{s+2} \leqslant Y_{s+2}} \cdots \sum_{X_{k}<p_{k} \leqslant Y_{k}} \exp \left(2 \pi i \alpha\left(p_{s+1} \ldots p_{k}\right)^{n}\right),
$$

имеем следуюшее равенство:

$$
S(\alpha)=\sum_{X_{s+1}<p_{s+1} \leqslant Y_{s+1}} S\left(\alpha ; p_{s+1}\right) .
$$

Возьмем $H=\left(2 k^{5}\right)^{4}, \quad H_{s+1}=\left(Y_{s+1}-X_{s+1}\right) H^{-1}$ и разделим промежуток $\left(X_{s+1}, Y_{s+1}\right]$ на $H$ промежутков $j_{r}$ следуюшего вида:

$$
j_{r}:\left(X_{s+1}+(r-1) H_{s+1}, X_{s+1}+r H_{s+1}\right], \quad r=1,2, \ldots, H .
$$

Тогда, соответственно этому разбиению, $S(\alpha)$ представится суммою $H$ слагаемых:

$$
S(\alpha)=\sum_{r=1}^{H} S_{r}(\alpha)
$$

где

$$
S_{r}(\alpha)=\sum_{p_{s+1} \in j_{r}} S\left(\alpha ; p_{s+1}\right) .
$$

5. Преобразуем $S^{k-s}(\alpha)$. Возведем обе части равенства (7) в степень $k-s$; получим

$$
S^{k-s}(\alpha)=\sum_{r_{1}=1}^{H} \cdots \sum_{r_{k-s}=1}^{H} S_{r_{1}}(\alpha) \ldots S_{r_{k-s}}(\alpha) .
$$

Все наборы чисел вида $\left(r_{1}, \ldots, r_{k-s}\right)$, где $1 \leqslant r_{1}, \ldots, r_{k-s} \leqslant H$, разобьем на классы $A$ и $B$ следуюшим образом: в класс $A$ отнесем такие наборы $\left(r_{1}, \ldots, r_{k-s}\right)$, в которых найдется $r_{j}$, отличное от всех остальных $r_{\nu}, j \neq \nu$, т.е. $r_{j} \neq r_{\nu}$ при 
$\nu \neq j$; в класс $B$ отнесем все остальные наборы. Сумму в правой части (9) по наборам $\left(r_{1}, \ldots, r_{k-s}\right)$ из класса $A$ обозначим $\Sigma_{1}$, а сумму по наборам $\left(r_{1}, \ldots, r_{k-s}\right)$ из класса $B$ обозначим $\Sigma_{2}$. Таким образом,

$$
\begin{aligned}
& \Sigma_{1}=\sum_{\left(r_{1}, \ldots, r_{k-s}\right) \in A} \ldots S_{r_{1}}(\alpha) \ldots S_{r_{k-s}}(\alpha), \\
& \Sigma_{2}=\sum_{\left(r_{1}, \ldots, r_{k-s}\right) \in B} \cdots S_{r_{1}}(\alpha) \ldots S_{r_{k-s}}(\alpha) .
\end{aligned}
$$

Равенство (9) перепишем так:

$$
S^{k-s}(\alpha)=\Sigma_{1}+\Sigma_{2} .
$$

6. Преобразуем слагаемые сумм $\Sigma_{1}$ и $\Sigma_{2}$. После перенумерации индексов наборов $\left(r_{1}, \ldots, r_{k-s}\right)$ слагаемые в $\Sigma_{1}$ имеют следуюший вид:

$$
S_{r_{1}}(\alpha) S_{r_{2}}(\alpha) \ldots S_{r_{k-s}}(\alpha),
$$

причем $r_{1} \neq r_{\nu}, \nu=2, \ldots, k-s$. Также после перенумерации индексов наборов $\left(r_{1}, \ldots, r_{k-s}\right)$ слагаемые в $\Sigma_{2}$ имеют следуюший вид:

$$
S_{r_{1}}^{\beta_{1}}(\alpha) \ldots S_{r_{t}}^{\beta_{t}}(\alpha)
$$

где $r_{\nu} \neq r_{j}, \nu \neq j, \beta_{1} \geqslant 2, \ldots, \beta_{t} \geqslant 2$, и, кроме того,

$$
\beta_{1}+\cdots+\beta_{t}=k-s .
$$

7. Пусть $\|A\|$ и $\|B\|$ - количество наборов $\left(r_{1}, \ldots, r_{k-s}\right)$ в классе $A$ и в классе $B$ соответственно. Для $\|A\|$ имеем тривиальную верхнюю оценку: $\|A\| \leqslant H^{k-s}$.

Оценим теперь сверху $\|B\|$. Так как числа $\beta_{1}, \ldots, \beta_{t}$ в (12) не меньше, чем 2 , то $t \leqslant 0.5(k-s)$. Пусть $r_{1}, \ldots, r_{t}, \beta_{1}, \ldots, \beta_{t}$ заданы. Это означает, что в наборе $\left(r_{1}, r_{2}, \ldots, r_{k-s}\right)$ число $r_{1}$ встречается $\beta_{1}$ раз, число $r_{2}$ встречается $\beta_{2}$ раза,$\ldots$, число $r_{t}$ встречается $\beta_{t}$ раз. Следовательно, на $k-s$ местах число $r_{1}$ занимает $\beta_{1}$ мест, что можно осуществить

$$
(k-s)(k-s-1) \ldots\left(k-s-\beta_{1}+1\right)<(k-s)^{\beta_{1}}
$$

способами, число $r_{2}$ занимает $\beta_{2}$ мест, что можно осуществить не более чем $(k-s)^{\beta_{2}}$ способами, $\ldots$, и, наконец, $r_{t}$ может занимать $\beta_{t}$ мест, что можно осушествить не более чем $(k-s)^{\beta_{t}}$ способами. Таким образом, количество слагаемых вида (11) при заданных $r_{1}, \ldots, r_{t}, \beta_{1}, \ldots, \beta_{t}$ не больше, чем

$$
(k-s) !(k-s)^{\beta_{1}+\cdots+\beta_{t}}<(k-s)^{2(k-s)} .
$$

Далее, если $r(t)$ - количество решений уравнения (11) в числах $\beta_{1}, \ldots, \beta_{t}$, то $r(t) \leqslant(k-s)^{t-1}$, поэтому для $\|B\|$ получаем такую верхнюю границу:

$$
\|B\| \leqslant \sum_{1 \leqslant t \leqslant 0.5(k-s)} H^{t} r(t)(k-s)^{2(k-s)} \leqslant 2(k-s)^{2.5(k-s)} H^{0.5(k-s)} .
$$


8. Перейдем в (10) к неравенствам. Возведем, далее, обе части этого неравенства в квадрат и воспользуемся неравенством Коши; получим

$$
\begin{aligned}
|S(\alpha)|^{2(k-s)} \leqslant & \left.2\|A\| \sum_{\left(r_{1}, \ldots, r_{k-s}\right) \in A} \ldots \sum_{r_{1}}(\alpha)\right|^{2} \ldots\left|S_{r_{k-s}}(\alpha)\right|^{2} \\
& +\left.2\|B\| \sum_{\left(r_{1}, \ldots, r_{k-s}\right) \in B} \ldots \sum_{r_{1}}(\alpha)\right|^{2} \ldots\left|S_{r_{k-s}}(\alpha)\right|^{2} .
\end{aligned}
$$

Слагаемые по $\left(r_{1}, \ldots, r_{k-s}\right)$ в правой части последнего неравенства преобразуем следуюшим образом. K произведениям второй кратной суммы применим неравенство между средним геометрическим и средним арифметическим. То же самое сделаем с произведениями первой кратной суммы, предварительно выделив первый множитель. Для слагаемых первой суммы получаем неравенство

$$
\begin{aligned}
& \left|S_{r_{1}}(\alpha)\right|^{2}\left|S_{r_{2}}(\alpha)\right|^{2} \ldots\left|S_{r_{k-s}}(\alpha)\right|^{2} \\
& \quad \leqslant \frac{1}{k-s-1}\left|S_{r_{1}}(\alpha)\right|^{2}\left(\left|S_{r_{2}}(\alpha)\right|^{2(k-s-1)}+\cdots+\left|S_{r_{k-s}}(\alpha)\right|^{2(k-s-1)}\right) .
\end{aligned}
$$

Отметим, что здесь $r_{1} \neq r_{j}, j=2, \ldots, k-s$.

Для слагаемых второй суммы получаем неравенство

$$
\left|S_{r_{1}}(\alpha)\right|^{2} \ldots\left|S_{r_{k-s}}(\alpha)\right|^{2} \leqslant \frac{1}{k-s}\left(\left|S_{r_{1}}(\alpha)\right|^{2(k-s)}+\cdots+\left|S_{r_{k-s}}(\alpha)\right|^{2(k-s)}\right) .
$$

Подставив (14) и (15) в (13), а затем (13) - в (6), приходим к неравенству

$$
I^{(s)} \leqslant 2\|A\|^{2} I_{1}^{(s)}+2\|B\|^{2} I_{2}^{(s)},
$$

где

$$
\begin{aligned}
I_{1}^{(s)} & =\int_{0}^{1}\left|S_{r_{1}}(\alpha)\right|^{2}\left|S_{r_{2}}(\alpha)\right|^{2(k-s-1)} d \alpha, \\
I_{2}^{(s)} & =\int_{0}^{1}\left|S_{r}(\alpha)\right|^{2(k-s)} d \alpha .
\end{aligned}
$$

В неравенстве (16) $r_{1}, r_{2}, r$ - фиксированные натуральные числа, причем $r_{1} \neq r_{2}$, $1 \leqslant r_{1}, r_{2}, r \leqslant H$, и, кроме того, такие, для которых интегралы $I_{1}^{(s)}$ и $I_{2}^{(s)}$ принимают свои максимальные значения. Еще заметим, что интеграл $I_{2}^{(s)}$ имеет такой же вид, что и $I^{(s)}$, только промежуток изменения переменной суммирования $p_{s+1}$ в сумме $S(\alpha)$, который имел вид

$$
X_{s+1}<p_{s+1} \leqslant Y_{s+1}
$$

заменился в сумме $S_{r}(\alpha)$ на более короткий промежуток вида

$$
X_{s+1}^{(1)}=X_{s+1}+(r-1) H_{s+1}<p_{s+1} \leqslant X_{s+1}+r H_{s+1}=Y_{s+1}^{(1)},
$$


длина которого равна

$$
H_{s+1}=Y_{s+1}^{(1)}-X_{s+1}^{(1)}=\left(Y_{s+1}-X_{s+1}\right) H^{-1}
$$

9. Преобразуем интеграл $I_{1}^{(s)}$. Так как

$$
S_{r_{2}}(\alpha)=\sum_{X_{s+1}^{(1)}<p_{s+1} \leqslant Y_{s+1}^{(1)}} S\left(\alpha ; p_{s+1}\right),
$$

то, переходя к неравенствам и применяя затем неравенство Гёльдера, найдем

$$
\begin{gathered}
\left|S_{r_{2}}(\alpha)\right|^{2(k-s-1)} \leqslant H_{s+1}^{2(k-s-1)-1} \sum_{X_{s+1}^{(1)}<p_{s+1} \leqslant Y_{s+1}^{(1)}}\left|S\left(\alpha ; p_{s+1}\right)\right|^{2(k-s-1)}, \\
I_{1}^{(s)} \leqslant H_{s+1}^{2(k-s-1)} \int_{0}^{1}\left|S_{r_{1}}(\alpha)\right|^{2}|S(\alpha ; p)|^{2(k-s-1)} d \alpha .
\end{gathered}
$$

В последнем интеграле буквой $p$ обозначено некоторое фиксированное простое число из промежутка $\left(X_{s+1}^{(1)}, Y_{s+1}^{(1)}\right]$, т.е.

$$
X_{s+1}^{(1)}=X_{s+1}+\left(r_{2}-1\right) H_{s+1}<p \leqslant X_{s+1}+r_{2} H_{s+1}=Y_{s+1}^{(1)}
$$

причем $r_{2} \neq r_{1}$ и число $p$ такое, при котором этот интеграл принимает максимальное значение.

10. Пусть

$$
I=\int_{0}^{1}\left|S_{r_{1}}(\alpha)\right|^{2}|S(\alpha ; p)|^{2(k-s-1)} d \alpha .
$$

Очевидно, что интеграл $I$ равен количеству решений следующего уравнения:

$$
x_{s+1}^{n}-y_{s+1}^{n}=p^{n}\left(x_{s+2}^{n}+\cdots+x_{k}^{n}-y_{s+2}^{n}-\cdots-y_{k}^{n}\right) \text {. }
$$

В этом уравнении неизвестные $x_{s+1}, y_{s+1}$ принимают значения чисел вида $p_{s+1} \ldots p_{k}$, а неизвестные $x_{s+2}, \ldots, x_{k}, y_{s+2}, \ldots, y_{k}$ принимают значения чисел вида $p_{s+2} \ldots p_{k}$, причем $p_{s+1}$ меняются в промежутке следуюшего вида:

$$
X_{s+1}+\left(r_{1}-1\right) H_{s+1}<p_{s+1} \leqslant X_{s+1}+r_{1} H_{s+1},
$$

а $p_{s+2}, \ldots, p_{k}$ меняются в промежутках вида

$$
X_{j}<p_{j} \leqslant Y_{j}, \quad j=s+2, \ldots, k \text {. }
$$

Так как $r_{2} \neq r_{1}$, то $p \neq p_{s+1}$.

Обозначим через $T$ количество решений в числах $x_{s+1}, y_{s+1}$ сравнения

$$
x_{s+1}^{n} \equiv y_{s+1}^{n} \quad\left(\bmod p^{n}\right) .
$$


Тогда для $I$ получаем такую оценку:

$$
I \leqslant T I_{1}
$$

где $I_{1}$ - количество решений уравнения

$$
x_{s+2}^{n}+\cdots+x_{k}^{n}=y_{s+2}^{n}+\cdots+y_{k}^{n}
$$

в числах $x_{j}, y_{j}, j=s+2, \ldots, k$, каждое из которых имеет вид $p_{s+2} \ldots p_{k}$. Легко видеть, что $I_{1}=I^{(s+1)}$.

11. Оценим сверху $T$. По определению неизвестные $x_{s+1}$ и $y_{s+1}$ имеют вид $p_{s+1} \ldots p_{k}$, причем $\left(p, x_{s+1}\right)=\left(p, y_{s+1}\right)=1$ и $X_{s+1}<p \leqslant Y_{s+1}$. Из верхних границ изменения $p_{s+1}, \ldots, p_{k}$ получаем оценки сверху для $x_{s+1}$ и $y_{s+1}$ :

$$
\max \left(x_{s+1}, y_{s+1}\right) \leqslant Y_{s+1} \ldots Y_{k}=N^{\left(1-\frac{1}{n}\right)^{s}-\left(1-\frac{1}{n}\right)^{k}} .
$$

В свою очередь, для $p^{n}$ выполняется такая нижняя оценка:

$$
p^{n}>X_{s+1}^{n}=\left(1-\frac{1}{4 k}\right)^{n} N^{\left(1-\frac{1}{n}\right)^{s}} .
$$

Из условий на $N, k, n, s$ легко следует неравенство

$$
N^{\left(1-\frac{1}{n}\right)^{s}-\left(1-\frac{1}{n}\right)^{k}}<\left(1-\frac{1}{4 k}\right)^{n} N^{\left(1-\frac{1}{n}\right)^{s}} .
$$

Тем самым неизвестные $x_{s+1}$ и $y_{s+1}$ сравнения (20) принимают значения некоторой части приведенной системы вычетов по модулю $p^{n}$. Поэтому при фиксированном $y_{s+1}$ сравнение (20) будет иметь не более чем $n$ решений, и, следовательно, для $T$ вьполняется оценка

$$
T \leqslant n\left(Y_{s+1}-X_{s+1}\right) \ldots\left(Y_{k}-X_{k}\right) .
$$

12. Соберем полученные оценки. Из (21), (22) находим

$$
I \leqslant n\left(Y_{s+1}-X_{s+1}\right) \ldots\left(Y_{k}-X_{k}\right) I^{(s+1)} .
$$

Из (19) следует неравенство

$$
I_{1}^{(s)} \leqslant H_{s+1}^{2(k-s-1)} I \leqslant n H_{s+1}^{2(k-s-1)}\left(Y_{s+1}-X_{s+1}\right) \ldots\left(Y_{k}-X_{k}\right) I^{(s+1)} .
$$

Наконец, из оценок $\|A\|,\|B\|$ и формул (16)-(19), (23) получаем первую основную оценку величины $I^{(s)}$ :

$$
\begin{aligned}
I^{(s)} \leqslant & 2 n H^{2(k-s)} H_{s+1}^{2(k-s-1)}\left(Y_{s+1}-X_{s+1}\right) \ldots\left(Y_{k}-X_{k}\right) I^{(s+1)} \\
& +2(k-s)^{5(k-s)} H^{k-s} I_{2}^{(s)} \\
= & 2 n H^{2(k-s)} H_{s+1}^{2(k-s-1)}\left(Y_{s+1}-X_{s+1}\right) L I^{(s+1)}+2(k-s)^{5(k-s)} H^{k-s} I_{2}^{(s)} .
\end{aligned}
$$


В этом неравенстве величины $H_{s+1}$ и $L$ соответственно равны:

$$
H_{s+1}=\left(Y_{s+1}-X_{s+1}\right) H^{-1}, \quad L=\left(Y_{s+2}-X_{s+2}\right) \ldots\left(Y_{k}-X_{k}\right),
$$

а интеграл $I_{2}^{(s)}$ имеет такой же вид, что и интеграл $I^{(s)}$, только величины $X_{s+1}$, $Y_{s+1}$, которые входят в определение $I^{(s)}$ (это есть гранищы изменения простых чисел $\left.p_{s+1}\right)$, заменились на новые, именно на $X_{s+1}^{(1)}, Y_{s+1}^{(1)}$, где

$$
X_{s+1}^{(1)}=X_{r+1}+(r-1) H_{s+1}, \quad Y_{s+1}^{(1)}=X_{s+1}+r X_{s+1}
$$

и $r$ - некоторое целое фиксированное число, $1 \leqslant r \leqslant H$.

13. Чтобы удобно было применять неравенство (24), введем новые обозначения и перепишем его в этих новых обозначениях. Положим

$$
\begin{gathered}
I^{(s)}=I_{2,0}^{(s)} ; \quad I_{2}^{(s)}=I_{2,1}^{(s)} ; \quad Y_{s+1}=Y_{s+1}^{(0)}, \quad X_{s+1}=X_{s+1}^{(0)} ; \\
H_{s+1}=H_{s+1}^{(0)}=\left(Y_{s+1}^{(0)}-X_{s+1}^{(0)}\right) H^{-1} ; \quad X_{s+1}^{(1)}=X_{s+1}^{(0)}+\left(r_{0}-1\right) H_{s+1}^{(0)}, \\
Y_{s+1}^{(1)}=X_{s+1}^{(0)}+r_{0} H_{s+1}^{(0)} .
\end{gathered}
$$

Тогда (24) перепишется так:

$$
\begin{aligned}
I_{2,0}^{(s)} \leqslant & 2 n H^{2(k-s)}\left(H_{s+1}^{(0)}\right)^{2(k-s-1)}\left(Y_{s+1}^{(0)}-X_{s+1}^{(0)}\right) L I^{(s+1)} \\
& +2(k-s)^{5(k-s)} H^{k-s} I_{2,1}^{(s)} .
\end{aligned}
$$

14. Определим теперь натуральное число $\mu$ неравенствами

$$
H^{\mu+2}<Y_{s+1}-X_{s+1} \leqslant H^{\mu+3} .
$$

Далее, определим при $j=0,1, \ldots, \mu$ параметры $X_{s+1}^{(j+1)}, Y_{s+1}^{(j+1)}, H_{s+1}^{(j)}$ равенствами

$$
\begin{gathered}
X_{s+1}^{(j+1)}=X_{s+1}^{(j)}+\left(r_{j}-1\right) H_{s+1}^{(j)}, \quad Y_{s+1}^{(j+1)}=X_{s+1}^{(j)}+r_{j} H_{s+1}^{(j)}, \\
H_{s+1}^{(j)}=\left(Y_{s+1}^{(j)}-X_{s+1}^{(j)}\right) H^{-1} .
\end{gathered}
$$

Здесь $r_{j}$ - некоторые целые числа (например, $r_{0}=r$ ), которые появляются в соответствующем итеративном процессе, $1 \leqslant r_{j} \leqslant H$. Тогда, повторяя рассуждения пп. $3-12$, приходим к неравенству, подобному (25):

$$
\begin{aligned}
I_{2, j}^{(s)} \leqslant & 2 n H^{2(k-s)}\left(H_{s+1}^{(j)}\right)^{2(k-s-1)}\left(Y_{s+1}^{(j)}-X_{s+1}^{(j)}\right) L I^{(s+1)} \\
& +2(k-s)^{5(k-s)} H^{k-s} I_{2, j+1}^{(s)} .
\end{aligned}
$$

15. Оценим сверху $I_{2, \mu+1}^{(s)}$. Этот интеграл тривиально оценим следующим обра30M:

$$
\begin{aligned}
I_{2, \mu+1}^{(s)} & =\int_{0}^{1}\left|\sum_{X_{s+1}^{(\mu+1)}<p_{s+1} \leqslant Y_{s+1}^{(\mu+1)}} S\left(\alpha ; p_{s+1}\right)\right|^{2(k-s)} d \alpha \\
& \leqslant\left(Y_{s+1}^{(\mu+1)}-X_{s+1}^{(\mu+1)}\right)^{2(k-s)} \int_{0}^{1}|S(\alpha ; p)|^{2(k-s)} d \alpha .
\end{aligned}
$$


Последний интеграл равен количеству решений такого уравнения:

$$
x_{s+1}^{n}+\cdots+x_{k}^{n}=y_{s+1}^{n}+\cdots+y_{k}^{n}
$$

причем неизвестные $x_{j}, y_{j}, j=s+1, \ldots, k$, принимают значения чисел вида $p_{s+2} \ldots p_{k}$. Поэтому, опять же применяя тривиальную оценку, находим

$$
\int_{0}^{1}|S(\alpha ; p)|^{2(k-s)} d \alpha \leqslant\left(Y_{s+1}-X_{s+1}\right)^{2} \ldots\left(Y_{k}-X_{k}\right)^{2} I^{(s+1)} .
$$

Из определения параметров $\mu, Y_{s+1}^{(j)}, X_{s+1}^{(j)}$ получаем

$$
Y_{s+1}^{(\mu+1)}-X_{s+1}^{(\mu+1)}=\left(Y_{s+1}-X_{s+1}\right) H^{-\mu-1} \leqslant H^{2}
$$

Поэтому из $(27)$ и $(28)$ приходим к нужной нам оценке $I_{2, \mu+1}^{(s)}$ :

$$
I_{2, \mu+1}^{(s)} \leqslant H^{4(k-s)}\left(Y_{s+1}-X_{s+1}\right)^{2} \ldots\left(Y_{k}-X_{k}\right)^{2} I^{(s+1)}
$$

16. Обозначим через $a$ множитель, стоящий перед $I_{2, j+1}^{(s)}$ в правой части неравенства (26), т.е.

$$
a=2(k-s)^{5(k-s)} H^{k-s} .
$$

Умножим обе части неравенства $(26)$ на $a^{j}$, получим

$$
a^{j} I_{2, j}^{(s)} \leqslant 2 n H^{2(k-s)} L I^{(s+1)} a^{j}\left(H_{s+1}^{(j)}\right)^{2(k-s-1)}+a^{j+1} I_{2, j+1}^{(s)} .
$$

Суммируя обе части (30) по $j=0,1, \ldots, \mu$, найдем

$$
\begin{aligned}
I_{2,0}^{(s)}+\sum_{j=1}^{\mu} a^{j} I_{2, j}^{(s)} \leqslant & 2 n H^{2(k-s)} L I^{(s+1)} \sum_{j=0}^{\mu} a^{j}\left(H_{s+1}^{(j)}\right)^{2(k-s-1)} \\
& +\sum_{j=1}^{\mu} a^{j} I_{2, j}^{(s)}+a^{\mu+1} I_{2, \mu+1}^{(s)} .
\end{aligned}
$$

Отсюда получаем такое неравенство (пользуемся тем, что $I_{2,0}^{(s)}=I^{(s)}$, и оценкой (29)):

$$
I^{(s)} \leqslant\left(2 n H^{2(k-s)} L V_{1}+V_{2}\right) I^{(s+1)}
$$

где

$$
\begin{aligned}
V_{1} & =\sum_{j=0}^{\mu} a^{j}\left(H_{s+1}^{(j)}\right)^{2(k-s-1)} \\
V_{2} & =a^{\mu+2} H^{4(k-s)}\left(Y_{s+1}-X_{s+1}\right)^{2} \ldots\left(Y_{k}-X_{k}\right)^{2} .
\end{aligned}
$$


17. Оценим сверху $V_{1}$. Так как

$$
\begin{gathered}
H=\left(2 k^{5}\right)^{4}, \quad a=2(k-s)^{5(k-s)} H^{k-s} \leqslant\left(2 k^{5}\right)^{k-s} H^{k-s}, \\
H_{s+1}^{(j)}=\left(Y_{s+1}-X_{s+1}\right) H^{-j-1}
\end{gathered}
$$

то слагаемые суммы $V_{1}$ оценим так:

$$
\begin{aligned}
& a^{j}\left(H_{s+1}^{(j)}\right)^{2(k-s-1)} \\
& \quad \leqslant\left(2 k^{5}\right)^{j(k-s)} H^{j(k-s)}\left(Y_{s+1}-X_{s+1}\right)^{2(k-s-1)} H^{-2(k-s-1)} H^{-j(2 k-2 s-2)} \\
& \quad=\left(Y_{s+1}-X_{s+1}\right)^{2(k-s-1)} H^{-2(k-s-1)}\left(\left(2 k^{5}\right)^{k-s} H^{-(k-s)+2}\right)^{j} \\
& \quad=\left(Y_{s+1}-X_{s+1}\right)^{2(k-s-1)} H^{-2(k-s-1)}\left(\left(2 k^{5}\right)^{-3(k-s)+8}\right)^{j} .
\end{aligned}
$$

Tак как $k-s \geqslant 2(n+3)$, то

$$
V_{1} \leqslant 2 H^{-2(k-s-1)}\left(Y_{s+1}-X_{s+1}\right)^{2(k-s-1)} .
$$

Докажем теперь, что вьполняются следующие неравенства:

$$
\begin{aligned}
& 2 n H^{2(k-s)} L \cdot 2 H^{-2(k-s-1)}\left(Y_{s+1}-X_{s+1}\right)^{2(k-s-1)} \\
& \quad \leqslant 4 n H^{2}\left(Y_{s+1}-X_{s+1}\right)^{2(k-s-1)} \prod_{j=2}^{k-s}\left(Y_{s+j}-X_{s+j}\right), \\
& a^{\mu+2} H^{4(k-s)}\left(Y_{s+1}-X_{s+1}\right)^{2} \ldots\left(Y_{k}-X_{k}\right)^{2} \\
& \quad \leqslant n H^{2}\left(Y_{s+1}-X_{s+1}\right)^{2(k-s-1)} \prod_{j=2}^{k-s}\left(Y_{s+j}-X_{s+j}\right) .
\end{aligned}
$$

Напомним, что здесь

$$
\begin{gathered}
L=\left(Y_{s+2}-X_{s+2}\right) \ldots\left(Y_{k}-X_{k}\right), \\
H^{\mu+2}<Y_{s+1}-X_{s+1} \leqslant H^{\mu+3}, \quad a=2(k-s)^{5(k-s)} H^{k-s} .
\end{gathered}
$$

Непосредственно видно, что соотношение (33) является равенством. Неравенство (34) после сокращения на общий множитель перейдет в такое:

$$
a^{\mu+2} H^{4(k-s)-2}\left(Y_{s+2}-X_{s+2}\right) \ldots\left(Y_{k}-X_{k}\right) \leqslant n\left(Y_{s+1}-X_{s+1}\right)^{2(k-s)-4} .
$$

Докажем более грубое неравенство, заменяя $a^{\mu+2}$ большей величиной:

$$
\begin{aligned}
& \left(2(k-s)^{5(k-s)}\right)^{\mu+2}\left(Y_{s+1}-X_{s+1}\right)^{k-s} H^{4(k-s)-2}\left(Y_{s+2}-X_{s+2}\right) \ldots\left(Y_{k}-X_{k}\right) \\
& \quad \leqslant n\left(Y_{s+1}-X_{s+1}\right)^{2(k-s)-4} .
\end{aligned}
$$

Так как $2 k^{5}=H^{\frac{1}{4}}$, то $\left(2(k-s)^{5}\right)^{k-s} \leqslant H^{\frac{k-s}{4}}$. Кроме того, $H^{\mu+2}<Y_{s+1}-X_{s+1}$. Поэтому будем доказывать еще более грубое неравенство

$$
\begin{gathered}
\left(Y_{s+1}-X_{s+1}\right)^{\frac{5}{4}(k-s)} H^{4(k-s)-2}\left(Y_{s+2}-X_{s+2}\right) \ldots\left(Y_{k}-X_{k}\right) \\
\leqslant n\left(Y_{s+1}-X_{s+1}\right)^{2(k-s)-4}
\end{gathered}
$$


или даже такое:

$$
H^{4(k-s)}\left(Y_{s+2}-X_{s+2}\right) \ldots\left(Y_{k}-X_{k}\right) \leqslant\left(Y_{s+1}-X_{s+1}\right)^{\frac{3}{4}(k-s)-4} .
$$

Заменяя $Y_{j}, X_{j}$ их значениями, находим

$$
\begin{aligned}
& Y_{j}-X_{j}=(4 k)^{-1} Y_{j}=(4 k)^{-1} N^{\frac{1}{n}\left(1-\frac{1}{n}\right)^{j-1}} \\
& \left(Y_{s+2}-X_{s+2}\right) \ldots\left(Y_{k}-X_{k}\right)=\left((4 k)^{-1}\right)^{k-s-1} N^{\frac{1}{n}}\left(\left(1-\frac{1}{n}\right)^{s+1}+\cdots+\left(1-\frac{1}{n}\right)^{k-1}\right) \text {, } \\
& \left(Y_{s+1}-X_{s+1}\right)^{\frac{3}{4}(k-s)-4}=\left((4 k)^{-1}\right)^{\frac{3}{4}(k-s)-4} N^{\frac{1}{n}\left(1-\frac{1}{n}\right)^{s}\left(\frac{3}{4}(k-s)-4\right)} .
\end{aligned}
$$

Поэтому (35) перепишется так:

$$
H^{4(k-s)}\left((4 k)^{-1}\right)^{\frac{k-s}{4}+3} \leqslant N^{\varkappa}
$$

где

$$
\begin{aligned}
\varkappa & =\frac{1}{n}\left(1-\frac{1}{n}\right)^{s}\left(\frac{3}{4}(k-s)-4\right)-\frac{1}{n}\left(\left(1-\frac{1}{n}\right)^{s+1}+\cdots+\left(1-\frac{1}{n}\right)^{k-1}\right) \\
& =\left(1-\frac{1}{n}\right)^{s}\left(\frac{3(k-s)}{4 n}-\frac{4}{n}-1+\frac{1}{n}+\left(1-\frac{1}{n}\right)^{k-s}\right) .
\end{aligned}
$$

Еще раз огрубим (36):

$$
H^{4(k-s)} \leqslant N^{\left(1-\frac{1}{n}\right)^{s}\left(\frac{3(k-s)}{4 n}-\frac{n+3}{n}\right)} .
$$

Отсюда получаем, что достаточно доказать неравенство

$$
H^{4} \leqslant N^{\left(1-\frac{1}{n}\right)^{s}\left(\frac{3}{4 n}-\frac{n+3}{n(k-s)}\right)} .
$$

Так как $k-s \geqslant 2(n+3)$, то докажем, что

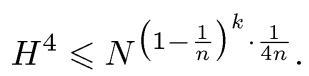

Вспоминая, что $N \asymp \log X, k \asymp \log ^{c} X, n \asymp \log ^{c} X, H=2 k^{5}$ и $0<c<1$, убеждаемся в справедливости неравенства (37) при $X \geqslant X_{1}(c)>0$.

Итак, неравенства (33) и (34) выполняются, что вместе с (32) дает нам вторую основную формулу:

$$
I^{(s)} \leqslant 5 n H^{2}\left(Y_{s+1}-X_{s+1}\right)^{2(k-s-1)} I^{(s+1)} \prod_{j=2}^{k-s}\left(Y_{s+j}-X_{s+j}\right) .
$$

Это есть неравенство (5) п. 2. 
18. Перемножая (5) при $s=0,1, \ldots, s_{1}$, получим

$$
\begin{aligned}
I^{(0)} \prod_{s=1}^{s_{1}} I^{(s)} \leqslant & I^{\left(s_{1}+1\right)}\left(\prod_{s=1}^{s_{1}} I^{(s)}\right)\left(5 n H^{2}\right)^{s_{1}+1} \\
& \times\left(\prod_{s=0}^{s_{1}}\left(Y_{s+1}-X_{s+1}\right)^{2(k-s-1)}\right)\left(\prod_{s=0}^{s_{1}} \prod_{j=2}^{k-s}\left(Y_{s+j}-X_{s+j}\right)\right) .
\end{aligned}
$$

Сокращая обе части этого неравенства на соответствующее произведение, приходим к оценке

$$
I^{(0)} \leqslant A B C D
$$

где

$$
\begin{array}{ll}
A=\left(5 n H^{2}\right)^{s_{1}+1}, & B=\prod_{s=0}^{s_{1}}\left(Y_{s+1}-X_{s+1}\right)^{2(k-s-1)}, \\
C=\prod_{s=0}^{s_{1}} \prod_{j=2}^{k-s}\left(Y_{s+j}-X_{s+j}\right), & D=I^{\left(s_{1}+1\right)} .
\end{array}
$$

Тривиальная оценка величины $I^{(s)}$ выглядит так (фиксируются все, кроме одной неизвестной):

$$
I^{(s)} \leqslant\left(\prod_{j=s+1}^{k}\left(Y_{j}-X_{j}\right)\right)^{2(k-s)-1}
$$

Поэтому

$$
D=I^{\left(s_{1}+1\right)} \leqslant\left(\prod_{j=s_{1}+2}^{k}\left(Y_{j}-X_{j}\right)\right)^{2\left(k-s_{1}-1\right)-1} .
$$

Так как $H=\left(2 k^{5}\right)^{4}, s_{1}=k-2(n+3) \geqslant 1, Y_{j}-X_{j}=(4 k)^{-1} N^{\frac{1}{n}\left(1-\frac{1}{n}\right)^{j-1}}$, для $A, B, C, D$ легко получаем такие неравенства:

$$
\begin{aligned}
A & <\left(1280 k^{41}\right)^{k-2 n-5}<4^{6(k-2 n-5)} k^{41(k-2 n-5)}, \\
B & =\prod_{s=0}^{s_{1}}(4 k)^{-2(k-s-1)} N^{\frac{2(k-s-1)}{n}\left(1-\frac{1}{n}\right)^{s}} \\
& =(4 k)^{-2 k\left(s_{1}+1\right)+\left(s_{1}+1\right)\left(s_{1}+2\right)} N^{2 k-2 n-(2 n+10)\left(1-\frac{1}{n}\right)^{s_{1}+1},} \\
C & =\prod_{s=0}^{s_{1}} \prod_{j=2}^{k-s}(4 k)^{-1} N^{\frac{1}{n}\left(1-\frac{1}{n}\right)^{s+j-1}} \\
& =\prod_{s=0}^{s_{1}}(4 k)^{-(k-s-1)} N^{\left(1-\frac{1}{n}\right)^{s+1}}-\left(1-\frac{1}{n}\right)^{k} \\
& =(4 k)^{-k\left(s_{1}+1\right)+\frac{1}{2}\left(s_{1}+1\right)\left(s_{1}+2\right)} N^{n-1-n\left(1-\frac{1}{n}\right)^{s_{1}+2}-\left(s_{1}+1\right)\left(1-\frac{1}{n}\right)^{k},}, \\
D & =\left(\prod_{j=s_{1}+2}^{k}(4 k)^{-1} N^{\frac{1}{n}\left(1-\frac{1}{n}\right)^{j-1}}\right)^{4 n+9}
\end{aligned}
$$




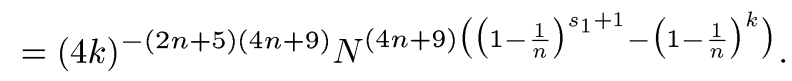

Из формулы (38) и найденных оценок величин $A, B, C, D$ приходим к окончательному неравенству для $I^{(0)}=I$ :

$$
I=I^{(0)}<a(k ; n) N^{2 k-n+\omega},
$$

где

$$
\begin{gathered}
a(k ; n)=(4 k)^{-(k-2 n-5)(1.5 k+n-41)-(2 n+5)(4 n+9)} \\
\omega=\omega(k ; n)=n\left(1-\frac{1}{n}\right)^{k-2 n-5}-(k+2 n+4)\left(1-\frac{1}{n}\right)^{k}-1 .
\end{gathered}
$$

Лемма доказана.

ЗАмЕчАнИЕ. Учитьвая условия $k>2(n+3)$ и $n \asymp \log ^{c} X$, для $a(k ; n)$ получаем простую форму оценки:

$$
a(k ; n)<\exp \left(-\frac{1}{8} k^{2} \log k\right) .
$$

\section{§3. Оценка тригонометрической суммы специального вида}

Применяя лемму 1 , оценим двойную тригонометрическую сумму $W(\alpha)$, определение которой будет дано в следующей лемме.

ЛЕмма 2. Пусть $\alpha-$ вещественное число, причем

$$
\alpha=\frac{a}{q}+\frac{\theta}{q^{2}}
$$

где $q \geqslant 3, \quad(a, q)=1, \quad|\theta| \leqslant 1$. Рассмотрим двойную тригонометрическую сум⿻м.

$$
W(\alpha)=\sum_{x \in V} \sum_{y \in V} \exp \left(2 \pi i \alpha m x^{n} y^{n}\right),
$$

где $x$ и $y$ пробегают значения $v$-чисел из $V$-множества, отвечающего параметрам $N, n, k ; m$ - целое положительное число. Тогда для $|W(\alpha)|$ справедлива следующая оценка:

$$
|W(\alpha)| \ll\|V\|^{2} \Delta_{0}
$$

¿əe

$$
\begin{aligned}
\Delta_{0} & =(m \log q)^{\frac{1}{4 k^{2}}}\left(N^{\omega_{1}} q^{-\frac{1}{4 k^{2}}}+N^{\omega_{2}} q^{\frac{1}{4 k^{2}}}\right) \log N \\
\omega_{1} & =-\frac{1}{2 k^{2}}+\frac{n}{2 k^{2}}\left(1-\frac{1}{n}\right)^{k-2 n-5}+\left(\frac{1}{2 k}-\frac{3 n+4}{2 k^{2}}\right)\left(1-\frac{1}{n}\right)^{k}, \\
\omega_{2} & =-\frac{n+1}{2 k^{2}}+\frac{n}{2 k^{2}}\left(1-\frac{1}{n}\right)^{k-2 n-5}+\left(\frac{1}{2 k}-\frac{n+2}{k^{2}}\right)\left(1-\frac{1}{n}\right)^{k} .
\end{aligned}
$$


ДокАЗАТЕльСтво. Пользуясь неравенством Гёльдера, последовательно получаем

$$
\begin{aligned}
|W(\alpha)|^{2 k} & \leqslant\left(\sum_{x \in V}\left|\sum_{y \in V} \exp \left(2 \pi i \alpha m x^{n} y^{n}\right)\right|\right)^{2 k} \\
& \leqslant\|V\|^{2 k-1} \sum_{x \in V}\left|\sum_{y \in V} \exp \left(2 \pi i \alpha m x^{n} y^{n}\right)\right|^{2 k} \\
& =\|V\|^{2 k-1} \sum_{x \in V} \sum_{\lambda} I_{k}(\lambda) \exp \left(2 \pi i \alpha m x^{n} \lambda\right),
\end{aligned}
$$

где символом $I_{k}(\lambda)$ обозначено количество решений уравнения

$$
y_{1}^{n}+\cdots+y_{k}^{n}=y_{k+1}^{n}+\cdots+y_{2 k}^{n}+\lambda
$$

в числах $y_{1}, \ldots, y_{2 k} \in V$. Так как для $y_{j}, 1 \leqslant j \leqslant 2 k$, выполняются неравенства $V_{1} \leqslant y_{j} \leqslant V_{2}$, то при $|\lambda|>\Lambda=k\left(V_{2}^{n}-V_{1}^{n}\right)$ число $I_{k}(\lambda)$ равно нулю. Поэтому в (39) считаем $|\lambda| \leqslant \Lambda$. Меняя в (39) порядки суммирования, приходим к следуюшей формуле:

$$
|W(\alpha)|^{2 k} \leqslant\|V\|^{2 k-1} \sum_{|\lambda| \leqslant \Lambda} I_{k}(\lambda)\left|\sum_{x \in V} \exp \left(2 \pi i \alpha m \lambda x^{n}\right)\right| .
$$

Возведем обе части этого неравенства в степень $2 k$ и опять применим неравенство Гёльдера; найдем

$$
\begin{aligned}
|W(\alpha)|^{4 k^{2}} & \leqslant\|V\|^{2 k(2 k-1)}\left(\sum_{|\lambda| \leqslant \Lambda} I_{k}(\lambda)\right)^{2 k-1} \sum_{|\lambda| \leqslant \Lambda} I_{k}(\lambda)\left|\sum_{x \in V} \exp \left(2 \pi i \alpha m \lambda x^{n}\right)\right|^{2 k} \\
& =\|V\|^{4 k^{2}-2 k} W_{1} W_{2}
\end{aligned}
$$

где

$$
\begin{aligned}
& W_{1}=\left(\sum_{|\lambda| \leqslant \Lambda} I_{k}(\lambda)\right)^{2 k-1}, \\
& W_{2}=\sum_{|\lambda| \leqslant \Lambda} I_{k}(\lambda)\left|\sum_{x \in V} \exp \left(2 \pi i \alpha m \lambda x^{n}\right)\right|^{2 k} .
\end{aligned}
$$

Оценим сверху каждую из выписанных вьше сумм $W_{1}, W_{2}$. Сумма величин $I_{k}(\lambda)$ по всем $\lambda$ равна количеству всех возможных наборов неизвестных $y_{1}, \ldots, y_{2 k}$ в уравнении (40), т.е.

$$
\sum_{|\lambda| \leqslant \Lambda} I_{k}(\lambda)=\|V\|^{2 k} ; \quad W_{1}=\|V\|^{4 k^{2}-2 k}
$$


Далее, так как

$$
\begin{aligned}
I_{k}(\lambda) & =\int_{0}^{1}\left|\sum_{x \in V} \exp \left(2 \pi i \alpha x^{n}\right)\right|^{2 k} \exp (-2 \pi i \alpha \lambda) d \alpha \\
& \leqslant \int_{0}^{1}\left|\sum_{x \in V} \exp \left(2 \pi i \alpha x^{n}\right)\right|^{2 k} d \alpha=I_{k}(0)
\end{aligned}
$$

то для $W_{2}$ получаем цепочку таких соотношений:

$$
\begin{aligned}
W_{2} & \leqslant I_{k}(0) \sum_{|\lambda| \leqslant \Lambda}\left|\sum_{x \in V} \exp \left(2 \pi i \alpha m \lambda x^{n}\right)\right|^{2 k} \\
& =I_{k}(0) \sum_{|\lambda| \leqslant \Lambda} \sum_{|\mu| \leqslant \Lambda} I_{k}(\mu) \exp (2 \pi i \alpha m \lambda \mu) \\
& \leqslant I_{k}(0) \sum_{|\mu| \leqslant \Lambda} I_{k}(\mu)\left|\sum_{|\lambda| \leqslant \Lambda} \exp (2 \pi i \alpha m \mu \lambda)\right| \\
& \leqslant I_{k}^{2}(0) \sum_{|\mu| \leqslant \Lambda}\left|\sum_{|\lambda| \leqslant \Lambda} \exp (2 \pi i \alpha m \mu \lambda)\right| .
\end{aligned}
$$

Воспользуемся теперь тем, что при любом вещественном числе $\beta$ справедливо неравенство

$$
\left|\sum_{|\lambda| \leqslant \Lambda} \exp (2 \pi i \beta \lambda)\right| \leqslant \min \left(2 \Lambda+1, \frac{1}{2\|\beta\|}\right) .
$$

Следовательно, из соотношений (44) для $W_{2}$ находим

$$
\begin{aligned}
W_{2} & \leqslant I_{k}^{2}(0) \sum_{|\mu| \leqslant \Lambda} \min \left(2 \Lambda+1, \frac{1}{2\|\alpha m \mu\|}\right) \\
& \leqslant I_{k}^{2}(0) \sum_{|\mu| \leqslant m \Lambda} \min \left(2 \Lambda+1, \frac{1}{2\|\alpha \mu\|}\right) .
\end{aligned}
$$

К сумме по $\mu$ применим известное неравенство (см. [8, с. 254] или [3, с. 94]); получим

$$
\begin{aligned}
W_{2} & \leqslant 6 I_{k}^{2}(0)\left(\frac{2 m \Lambda+1}{q}+1\right)(2 \Lambda+1+q \log q) \\
& \leqslant 36 I_{k}^{2}(0) \frac{m}{q}(\Lambda+q)^{2} \log q
\end{aligned}
$$

Замечая, что $I_{k}(0)=I$, где $I$ определено и оценено в лемме 1 , из (41)-(45) последовательно находим

$$
\begin{gathered}
|W(\alpha)|^{4 k^{2}} \leqslant 36\|V\|^{8 k^{2}-4 k} I^{2} \frac{m}{q}(\Lambda+q)^{2} \log q \\
|W(\alpha)| \leqslant\|V\|^{2} \Delta \\
\Delta=\left(\frac{36 m}{q}(\Lambda+q)^{2} \log q\right)^{\frac{1}{4 k^{2}}} I^{\frac{1}{2 k^{2}}}\|V\|^{-\frac{1}{k}}
\end{gathered}
$$


Чтобы получить нужную оценку $\Delta$, воспользуемся оценками сверху величин $\Lambda, I$ и нижней оценкой $\|V\|$ :

$$
\begin{gathered}
\Lambda<k V_{2}^{n}=k N^{n-n\left(1-\frac{1}{n}\right)^{k}} \\
I<a(k ; n) N^{2 k-n+\omega}, \\
\|V\| \geqslant\left(\frac{n}{8 k \log N}\right)^{k}\left(1+\frac{1}{n-1}\right)^{\frac{k(k-1)}{2}} N^{1-\left(1-\frac{1}{n}\right)^{k} .}
\end{gathered}
$$

Вводя знак Виноградова « и учитывая замечание к лемме 1 , находим

$$
\begin{aligned}
& I^{\frac{1}{2 k^{2}}}\|V\|^{-\frac{1}{k}} \ll \frac{k \log N}{n} N^{\frac{1}{k}-\frac{n}{2 k^{2}}+\frac{\omega}{2 k^{2}}-\frac{1}{k}+\frac{1}{k}\left(1-\frac{1}{n}\right)^{k}} \\
& \ll(\log N) N^{-\frac{n}{2 k^{2}}-\frac{1}{2 k^{2}}+\frac{n}{2 k^{2}}\left(1-\frac{1}{n}\right)^{k-2 n-5}+\left(\frac{1}{2 k}-\frac{n+2}{k^{2}}\right)\left(1-\frac{1}{n}\right)^{k}}, \\
& \Lambda^{\frac{1}{2 k}} q^{-\frac{1}{4 k^{2}}} \ll N^{\frac{n}{2 k^{2}}-\frac{n}{2 k^{2}}\left(1-\frac{1}{n}\right)^{k}} q^{-\frac{1}{4 k^{2}}}, \\
& \Delta \ll \Delta_{1} \ll(m \log q)^{\frac{1}{4 k^{2}}}\left(N^{\omega_{1}} q^{-\frac{1}{4 k^{2}}}+N^{\omega_{2}} q^{\frac{1}{4 k^{2}}}\right) \log N,
\end{aligned}
$$

где

$$
\begin{aligned}
\omega_{1}= & \frac{n}{2 k^{2}}\left(1-\frac{1}{n}\right)^{k-2 n-5}-\frac{1}{2 k^{2}}+\frac{1}{2 k}\left(1-\frac{1}{n}\right)^{k} \\
& -\frac{n+2}{k^{2}}\left(1-\frac{1}{n}\right)^{k}-\frac{n}{2 k^{2}}\left(1-\frac{1}{n}\right)^{k} \\
= & \frac{n}{2 k^{2}}\left(1-\frac{1}{n}\right)^{k-2 n-5}-\frac{1}{2 k^{2}}+\left(\frac{1}{2 k}-\frac{3 n+4}{2 k^{2}}\right)\left(1-\frac{1}{n}\right)^{k}, \\
\omega_{2}= & -\frac{n+1}{2 k^{2}}+\frac{n}{2 k^{2}}\left(1-\frac{1}{n}\right)^{k-2 n-5}+\left(\frac{1}{2 k}-\frac{n+2}{k^{2}}\right)\left(1-\frac{1}{n}\right)^{k} .
\end{aligned}
$$

Отсюда и из соотношения (46) следует утверждение леммы.

ЗАмЕчАниЕ. Чтобы оценка леммы 2 была не тривиальной, необходимо выполнение неравенств

$$
N^{4 k^{2} \omega_{1}}<q<N^{-4 k^{2} \omega_{2}} .
$$

\section{§4. Доказательство теоремы}

1. Прежде всего заметим, что при $X \geqslant X_{1}(c)>0$ выполняется равенство $\left[\log ^{c} X\right]=\left[\log ^{c} x\right]$ либо при всех $x$ из промежутка $[0.5 X, X]$, либо при всех $x$ из промежутка $[X, 2 X]$. Действительно, если $\left\{\log ^{c} X\right\}>4 \log ^{-1+c} X$, то

$$
\begin{aligned}
(\log 0.5 X)^{c} & =\left(\log ^{c} X\right) \cdot\left(1-\frac{\log 2}{\log X}\right)^{c} \\
& =\log ^{c} X-c \frac{\log 2}{\log X} \log ^{c} X+\cdots=\left[\log ^{c} X\right]+\left\{\log ^{c} X\right\}-\cdots>\left[\log ^{c} X\right] .
\end{aligned}
$$


Следовательно, при $0.5 X \leqslant x \leqslant X$ имеем

$$
\left[\log ^{c} X\right]<(\log 0.5 X)^{c} \leqslant \log ^{c} x \leqslant \log ^{c} X<\left[\log ^{c} X\right]+1,
$$

и при всех $x \in[0.5 X, X]$

$$
\left[\log ^{c} x\right]=\left[\log ^{c} X\right]
$$

Если же $\left\{\log ^{c} X\right\} \leqslant 4 \log ^{-1+c} X$, то

$$
\begin{aligned}
(\log 2 X)^{c} & =\left(\log ^{c} X\right)\left(1+\frac{\log 2}{\log X}\right)^{c}=\log ^{c} X+c(\log 2) \log ^{c-1} X+\cdots \\
& =\left[\log ^{c} X\right]+\left\{\log ^{c} X\right\}+c(\log 2) \log ^{c-1} X+\cdots<\left[\log ^{c} X\right]+1 .
\end{aligned}
$$

Следовательно, при $X \leqslant x \leqslant 2 X$

$$
\left[\log ^{c} X\right] \leqslant \log ^{c} X \leqslant \log ^{c} x \leqslant(\log 2 X)^{c}<\left[\log ^{c} X\right]+1,
$$

и при всех $x \in[X, 2 X]$ имеем

$$
\left[\log ^{c} x\right]=\left[\log ^{c} X\right]
$$

Поэтому, никак не ограничивая обшности, будем считать $X$ таким, что $\left[\log ^{c} x\right]=$ $\left[\log ^{c} X\right]$ при всех $x$ из промежутка $[0.5 X, X]$.

2. Полагая $n=\left[\log ^{c} X\right], k=10 n$, определим число $N$ равенством

$$
X=N^{2-2\left(1-\frac{1}{n}\right)^{k}}
$$

Пусть $V=V(N ; n, k)$ - множество $v$-чисел, отвечающее параметрам $N, n, k$. При $0 \leqslant a<b \leqslant 1$ символом $K=K(X ; a, b)$ обозначим количество чисел $z=x y$, где $x$ и $y$ пробегают независимо друг от друга значения $v$-чисел множества $V$, таких, что

$$
a \leqslant\left\{\alpha \exp \left(\left[\log ^{c} z\right] \log z\right)\right\}<b .
$$

3. Пусть $r=2[\log X], \Delta=N^{-\frac{n}{k^{2}}}$, числа $a_{1}$ и $b_{1}$ таковы, что $0 \leqslant a_{1}<b_{1} \leqslant 1$, $\Delta \leqslant b_{1}-a_{1} \leqslant 1-\Delta$, и $\psi(x)$ - "стаканчик" Виноградова, отвечающий параметрам $r, \Delta, a_{1}, b_{1}$ (см., например, [3, c. 14]). Найдем асимптотику следующей суммы $K_{1}$ :

$$
K_{1}=K_{1}\left(X ; a_{1}, b_{1}\right)=\sum_{z} \psi\left(\alpha \exp \left(\left[\log ^{c} z\right] \log z\right)\right) .
$$

4. Прежде всего функция $\psi(x)$ разлагается в такой ряд Фурье:

$$
\psi(x)=b_{1}-a_{1}+\sum_{m \neq 0} g(m) \exp (2 \pi i m x),
$$

где

$$
|g(m)| \leqslant \min \left(b_{1}-a_{1}, \frac{1}{\pi|m|}, \frac{1}{\pi|m|}\left(\frac{r}{\pi|m| \Delta}\right)^{r}\right) .
$$


Пользуясь при $|m|>m_{1}=2 r \Delta^{-1}$ третьей оценкой $|g(m)|$, получаем

$$
\begin{aligned}
\left|\sum_{|m|>m_{1}} g(m) \exp (2 \pi i m x)\right| & \leqslant \frac{2}{\pi}\left(\frac{r}{\pi \Delta}\right)^{r} \sum_{m>m_{1}} \frac{1}{m^{r+1}} \\
& <\frac{1}{\pi}\left(\frac{r}{\pi \Delta m_{1}}\right)^{r}<X^{-3}
\end{aligned}
$$

Поэтому для $K_{1}$ справедлива следующая формула:

$$
K_{1}=\left(b_{1}-a_{1}\right)\|V\|^{2}+\sum_{0<|m| \leqslant m_{1}} g(m) S(m)+\theta\|V\|^{2} X^{-3},
$$

где

$$
\begin{aligned}
S(m) & =\sum_{z} \exp (2 \pi i m f(z)), \\
f(z) & =\alpha \exp \left(\left[\log ^{c} z\right] \log z\right) .
\end{aligned}
$$

5. Так как $z=x y, x \in V, y \in V$, то нижняя и верхняя границы изменения $z$ легко находятся:

$$
0.5 X \leqslant\left(1-\frac{1}{4 k}\right)^{2 k} N^{2-2\left(1-\frac{1}{n}\right)^{k}} \leqslant z \leqslant N^{2-2\left(1-\frac{1}{n}\right)^{k}}=X .
$$

Поэтому при всех значениях $z$ выполняется равенство

$$
\left[\log ^{c} z\right]=\left[\log ^{c} X\right]=n,
$$

и, следовательно, $f(z)=\alpha z^{n}=\alpha x^{n} y^{n}$. Таким образом, $S(m)$ есть сумма из леммы 2.

6. Так как $\alpha$-вешественное алгебраическое число степени, не меньшей 2 , то $\alpha$ представляется бесконечной цепной дробью. Пусть $P_{\nu}$ и $Q_{\nu}$ - числитель и знаменатель $\nu$-й подходящей дроби $\alpha, \nu=1,2, \ldots$ Известно, что $\left(P_{\nu}, Q_{\nu}\right)=1$ и, кроме того,

$$
\left|\alpha-\frac{P_{\nu}}{Q_{\nu}}\right|<\frac{1}{Q_{\nu} Q_{\nu+1}} .
$$

По теореме Туэ-Зигеля-Рота для любого $\varepsilon>0$ существует $c_{5}=c_{5}(\alpha ; \varepsilon)>0$ такое, что для любой рациональной дроби $P_{\nu} / Q_{\nu},\left(P_{\nu}, Q_{\nu}\right)=1$, выполняется неравенство

$$
\left|\alpha-\frac{P_{\nu}}{Q_{\nu}}\right| \geqslant \frac{c_{5}}{Q_{\nu}^{2+\varepsilon}}
$$

Из неравенств (48) и (49) находим оценку для $Q_{\nu+1}$ :

$$
Q_{\nu}<Q_{\nu+1} \leqslant c_{6} Q_{\nu}^{1+\varepsilon} .
$$

Возьмем $\varepsilon=0,01, c_{6}=c_{6}(\alpha)>0$ и определим натуральное число $\nu$ неравенствами

$$
Q_{\nu}<N^{n-n\left(1-\frac{1}{n}\right)^{k}} \leqslant Q_{\nu+1} .
$$


Обозначая через $q$ число $Q_{\nu}$, а через $a-$ число $P_{\nu}$, получим

$$
\alpha=\frac{a}{q}+\frac{\theta}{q^{2}}, \quad(a, q)=1, \quad|\theta| \leqslant 1
$$

Из неравенств (50) и (51) для $q$ находим нужные нам верхнюю и нижнюю оценки:

$$
c_{7} N^{\frac{100}{101} \varkappa} \leqslant q \leqslant N^{\varkappa}, \quad \varkappa=n-n\left(1-\frac{1}{n}\right)^{k} .
$$

7. K сумме $S(m)$ применим лемму 2 , получим

$$
|S(m)| \ll(m \log q)^{\frac{1}{4 k^{2}}}\left(N^{\omega_{1}} q^{-\frac{1}{4 k^{2}}}+N^{\omega_{2}} q^{\frac{1}{4 k^{2}}}\right)\|V\|^{2} \log N .
$$

Из определения величин $m_{1}=2 r \Delta^{-1}, \Delta, \omega_{1}, \omega_{2}, k, n, q$ и оценки (52) после несложных вычислений приходим к следуюшим неравенствам:

$$
\begin{aligned}
(m \log q)^{\frac{1}{4 k^{2}}} & \leqslant\left(m_{1} n \log N\right)^{\frac{1}{4 k^{2}}} \ll\left(\Delta^{-1} \log ^{3} X\right)^{\frac{1}{4 k^{2}}} \\
& =\left(N^{\frac{n}{k^{2}}} \log ^{3} X\right)^{\frac{1}{4 k^{2}}} \ll N^{\frac{n}{4 k^{4}}}, \\
N^{\omega_{1}} q^{-\frac{1}{4 k^{2}}} & \ll N^{\omega_{1}-\frac{1}{4 k^{2}}\left(1-\frac{1}{101}\right)\left(n-n\left(1-\frac{1}{n}\right)^{k}\right)} \leqslant N^{-\frac{n}{8 k^{2}}}, \\
N^{\omega_{2}} q^{\frac{1}{4 k^{2}}} & \leqslant N^{\omega_{2}+\frac{1}{4 k^{2}}\left(n-n\left(1-\frac{1}{n}\right)^{k}\right)} \leqslant N^{-\frac{n}{8 k^{2}}}, \\
N^{\frac{n}{4 k^{4}}} \cdot N^{-\frac{n}{8 k^{2}}} & \leqslant N^{-\frac{n}{9 k^{2}}} \\
|S(m)| & \ll N^{-\frac{n}{9 k^{2}}}\|V\|^{2} \log N .
\end{aligned}
$$

8. Оценивая при $0<|m| \leqslant m_{1}$ коэффициенты $g(m)$ ряда Фурье функции $\psi(x)$ величиной $1 / \pi|m|$, из (47) и (53) находим асимптотическую формулу для $K_{1}$ :

$$
\begin{aligned}
K_{1} & =\left(b_{1}-a_{1}\right)\|V\|^{2}+\theta_{1} c_{8}\|V\|^{2} N^{-\frac{n}{9 k^{2}}} \log N+\theta_{1}\|V\|^{2} X^{-3} \\
& =\left(b_{1}-a_{1}\right)\|V\|^{2}+\theta_{2}\|V\|^{2} N^{-\frac{n}{10 k^{2}}} .
\end{aligned}
$$

9. Теперь применим лемму В из [3, с. 16]. Полагая в ней $r=2[\log X], \Delta=N^{-\frac{n}{k^{2}}}$, $\delta_{s}=\left\{\alpha \exp \left(\left[\log ^{c} z\right] \log z\right)\right\}, \quad s=1,2, \ldots,\|V\|^{2}$, и учитьвая асимптотическую формулу для $K_{1}=K_{1}\left(X ; a_{1}, b_{1}\right)$, в силу п. а) этой леммы получаем

$$
\begin{aligned}
K & =K(X ; a, b)=(b-a)\|V\|^{2}+O\left(\|V\|^{2} N^{-\frac{n}{10 k^{2}}}\right)+O\left(\|V\|^{2} N^{-\frac{n}{k^{2}}}\right) \\
& =(b-a)\|V\|^{2}+O\left(\|V\|^{2} N^{-\frac{n}{10 k^{2}}}\right) .
\end{aligned}
$$

Отсюда следует, что при любых $a$ и $b, 0 \leqslant a<b \leqslant 1$,

$$
b-a \gg N^{-\frac{n}{10 k^{2}}}=\exp \left(-c_{1} \log ^{1-c} X\right),
$$

для $K$ выполняется нижняя оценка:

$$
K \gg(b-a)\|V\|^{2}
$$


Как уже отмечалось вьше, количество решений уравнения $x y=z, x \in V, y \in V$, не превосходит $2^{k}$. Поэтому количество натуральных чисел $z$, не превосходящих $X$ и таких, что

$$
a \leqslant\left\{\alpha \exp \left(\left[\log ^{c} z\right] \log z\right)\right\}<b,
$$

не меньше, чем

$$
\begin{aligned}
c_{8} 2^{-k}(b-a)\|V\|^{2} \geqslant & c_{9} 2^{-10 n} N^{-\frac{n}{10 k^{2}}}\left(\frac{8 k \log N}{n}\right)^{-2 k} \\
& \times\left(1+\frac{1}{n-1}\right)^{k(k-1)} N^{2-2\left(1-\frac{1}{n}\right)^{k}} \\
\geqslant & X \exp \left(-c_{10}\left(\log ^{1-c} X+\log ^{c} X \log \log X\right)\right) .
\end{aligned}
$$

В частности, отсюда получаем, что при любом вещественном числе $\xi$ количество решений в натуральных числах $z$ системы неравенств $z \leqslant X$,

$$
\|\xi-f(z)\| \leqslant \exp \left(-c_{1} \log ^{1-c} X\right)
$$

не меньше, чем

$$
X \exp \left(-c_{10}\left(\log ^{1-c} X+\log ^{c} X \log \log X\right)\right),
$$

и, следовательно,

$$
\min _{z \leqslant X}\|\xi-f(z)\| \leqslant \exp \left(-c_{1} \log ^{1-c} X\right)
$$

Теорема доказана.

ЗАмЕчАниЕ. Утверждение теоремы остается в силе, если $\alpha$ - иррациональное число с ограниченными неполными частными.

\section{Список литературы}

1. Карачуба A. А. Оценки тригонометрических сумм методом И. М. Виноградова и их применения // Тр. МИАН. 1971. Т. 112. С. 241-255.

2. Карацуба A. А. Основы аналитической теории чисел. М.: Наука, 1975.

3. Карацуба A. А. Основы аналитической теории чисел. 2-е изд. М.: Наука, 1983.

4. Hardy G. H., Littlewood J. E. Some problems of Diophantine approximation // Internat. Congress of Math. Cambridge. 1912. V. 1. P. 223-229.

5. Weyl H. Über die Gleichverteilung von Zahlen mod Eins // Math. Ann. 1916. V. 77. P. 313-352.

6. Виноградов И. М. Аналитическое доказательство теоремы о распределении дробных частей целого многочлена // Изв. АН СССР. 6 серия. 1927. Т. 21. С. 567-578.

7. Виноградов И. М. О приближениях посредством рациональных дробей, имеющих знаменателем точную степень // ДАН СССР. 1935. Т. 2. С. 1-5.

8. Виноградов И. М. Избранные труды. М.: Изд-во АН СССР, 1952.

9. Карацуба А. А. Тригонометрические суммы и их применения // Тр. МКМ. Ванкувер. 1975. T. 1. C. $365-368$.

10. Карачуба A.A. О некоторых арифметических задачах с числами, имеющими малые простые делители // Acta Arith. 1975. Т. 27. С. 489-492.

11. Карачуба A. A. О функции $G(n)$ в проблеме Варинга // Изв. АН СССР. Сер. матем. 1985. T. 49. № 5. C. 935-947.

12. Карацуба А. А. Об одном диофантовом неравенстве // Acta Arith. 1989. T. LIII. C. 309-324. 